\title{
Analysis of neuropsychological functioning in patients with chronic fatigue syndrome
}

\author{
Jordan Grafman, Vicki Schwartz, Janet K Dale, Marten Scheffers, Christine Houser, \\ Stephen E Straus
}

\begin{abstract}
Memory impairment dominates the cognitive complaints of patients with chronic fatigue syndrome (CFS). Twenty CFS patients were available for studies with a clinical and experimental battery composed of memory and cognitive tests. The results on objective testing indicated that the CFS patients had some mild memory impairment, but only on tasks requiring conceptually driven encoding and retrieval processes. There were no associations between the nature of the precipitating illness, self ratings of fatigue, physical findings, or laboratory determination and objective memory performance or self report of memory functioning. These generally negative results indicate that memory impairment in CFS patients is typically mild and involves memory processes that participate in conceptualising information.
\end{abstract}

(F Neurol Neurosurg Psychiatry 1993;56:684-689)

Medical professionals are frequently required to assess and manage patients who complain of chronic fatigue. When associated with pain, sore throat, feverishness, tender lymph nodes, decreased concentration, memory problems, and depressed mood in the absence of a discernible cause, the symptom complex is termed the chronic fatigue syndrome (CFS). ${ }^{1}$ The lack of uniform physical or laboratory abnormalities in patients with CFS dictates that the diagnosis is one of exclusion, and that treatment is empirical. ${ }^{23}$ In addition, there is a striking overall similarity between CFS and the condition long known as neurasthenia. ${ }^{4-6}$

Depression is a prominent feature in many patients with CFS. While in some it predates the illness and may contribute to this syndrome's immune, somatic, and cognitive features, ${ }^{7-13}$ in others, the depressive symptoms may represent reactions to the debilitating illness and/or be a co-morbid expression of constitutional factors that ultimately make people susceptible to delayed recovery from infections or other acutely stressful events. ${ }^{7-91213}$ This combination of factors has fuelled an unresolved debate as to whether CFS is the reflection of an underlying psychiatric disturbance or, instead, is the result of an ongoing disorder with an infectious or immune origin. ${ }^{14-18}$ Regardless of the outcome of this debate, qualitative and quan- titative characterisation of the clinical features of CFS would permit better patient classification and management.

Among the least studied features of CFS are the common complaints of deficits in memory, cognition, and mood. ${ }^{19}$ The goals of the present study were to characterise the dimensions of these neuropsychological problems and to determine the degree to which they correlate with other clinical and laboratory measures.

In a pilot study, we administered retrospective and current period surveys to 54 patients who met Center for Disease Control criteria for CFS and who were participating in ongoing research studies at the National Institutes of Health. These patients underwent medical and laboratory evaluations and completed self rating forms regarding mood and cognitive functions. ${ }^{20}$ The results showed that CFS patients report many symptoms that are characteristic of depression, and that the prevalence and severity of depressive complaints reflect the degree of overall illness and its cognitive features, especially those regarding memory, an observation made in an earlier controlled therapeutic trial in patients such as these. ${ }^{2} \mathrm{We}$ extended these earlier observations by a detailed assessment of cognitive complaints in CFS patients and by analysis of their relationship with depressive complaints and severity of illness. Our results indicated that complaints of memory impairment dominate the current and past reports of patients with CFS. Moreover, the severity levels of cognitive and mood problems recalled during the worst period of illness correlated highly with the current cognitive and mood levels. For both the current and past periods, the level of depression correlated directly with the number and severity of memory complaints. Importantly, neither the current or recalled complaints of cognitive and mood problems could be predicted on the basis of any other clinical or laboratory data accrued.

We, therefore, decided to examine CFS patients with a battery of cognitive and memory tests to determine whether objective memory and cognitive deficits could be demonstrated in the light of the survey results.

\section{Methods}

SUBJECTS

The first 20 of the survey respondents who subsequently travelled to the NIH for further biological studies were neuropsychologically 
evaluated. They were compared with 17 healthy control subjects matched for age and education (see table 1). This subset of patients was similar in all respects to the larger group of patients we surveyed.

\section{MEASURES}

A set of measures was chosen that would evaluate objectively the cognitive complaints of CFS patients based on the pilot survey study. While we focused on memory measures, tests of planning, reaction time and intellectual capacity were also included in the battery. Two "Tower" planning tasks were selected because they represent different difficulty levels in planning. Thus, this battery not only measures those domains of cognition noted by CFS patients to be taxing, but includes measures known to be sensitive to "subcortical dementia" such as time perception, response speed, and planning. The following battery of tests and scales was administered over a total of eight hours on two consecutive days of study. A more detailed description of these tests, except where noted, is available in Lezak. ${ }^{21}$

All patients and controls demonstrated excellent effort and motivation during the evaluation. All of the patients remarked that the examination was tiring for them. Some patients complained of debilitating fatigue only on the day following the completion of the evaluation.

\section{GENERAL INTELLECTUAL PERFORMANCE}

1. Wechsler adult intelligence scale revised

This is a standard intelligence test composed of 11 subtests assessing verbal and non-verbal abilities. Both scale and subtest scores are reported.

\section{TIMING AND REACTION TIME TASKS \\ 1. Simple reaction time}

Subjects were instructed to press the space bar on a computer keyboard as quickly as possible every time a stimulus appeared on a computer monitor. There were a total of 21 trials on this task.

\section{Serial reaction time test ${ }^{22}$}

Subjects were instructed to press one of four keys that were spatially aligned with one of four positions on a computer monitor. Stimuli would appear at one of the four positions, one at a time, and subjects were to press the key that was underneath the position where the stimulus would appear as quickly as possible. There were seven blocks of 100 trials each. The first two blocks were composed of random presentations, the next four blocks were composed of 10 repetitions

Table 1 Subject characteristics

\begin{tabular}{lll}
\hline & Controls $(N=17)$ & CFS patients $(N=20)$ \\
\hline Age (years) & $38 \cdot 1(8 \cdot 9)$ & $37 \cdot 2(9 \cdot 0)$ \\
Education (years) & $15 \cdot 9(2 \cdot 7)$ & $15 \cdot 5(2 \cdot 2)$ \\
Handedness & $100 \%$ Right Handed & $100 \%$ Right Handed \\
Sex & 9 Women/8 Men & 12 Women $/ 8$ Men \\
\hline (Means and standard deviations are shown.)
\end{tabular}

of a fixed sequence of 10 stimuli, and the last block was another random presentation. Familiarity with the task itself should be reflected by a slight decrease in reaction times (RT) across the first two blocks. The further expected decline in RT across blocks three to six indicates the procedural learning of the repeated sequence. The expected increase in RT on block seven (composed of random trials) compared with block six (the last repeated trial block) is an indication of visuomotor procedural learning (the difference score that we use subtracts the RT of block seven from block six). The RT for blocks one and two also represents a choice-RT measure independent of procedural learning.

\section{Time wall}

Subjects viewed a block falling from the top of a computer monitor at a steady velocity. Half way down the screen the block disappeared from view behind an opaque mask. Subjects had to press a key when they believed the block had reached the bottom of the screen. This task measures time perception based on encoding of the speed of a visual stimulus.

\section{Time clock}

Subjects saw a circle on a computer monitor and were asked to press a key once every second for 60 keypresses. After each keypress, the subject saw a clock hand move $1 / 60$ of the distance around the circumference of the circle in a clockwise direction. This task measures elapsed time estimation when the target time interval ( $\sim 1$ second) is known.

\section{PROBLEM SOLVING AND PLANNING}

\section{Tower of London ${ }^{23}$}

On this simple task of planning and procedural knowledge, subjects were shown a collection of three coloured discs placed upon a set of different sized pegs in a certain pattern defined as the initial state. They were then shown a card indicating the final state that the discs should be in. Subjects were then told to move the discs one at a time until they reached the final state. Only one disc could be moved at a time, there was a limit on how many discs could be placed on the pegs, and there was a time limit of two minutes by the end of which a move had to be made. If a violation of any of these rules was made, the subject was allowed to start over again. A total of three attempts per problem was allowed. Response times were recorded per move.

\section{Tower of $\mathrm{Hanoi}^{23}$}

This is a more demanding task than the Tower of London but measures similar planning and procedural knowledge skills. Subjects were shown the graphic image of a collection of five discs placed upon a set of pegs on a computer screen. From this initial state, subjects were asked to use the numerical keys on the keyboard to shift discs to different pegs in order to reach a final state. Subjects had to solve the problem within two 
minutes. The number correct out of nine problems was recorded. Each problem had a different initial state but a constant final state.

\section{Twenty questions}

Subjects had to identify the correct object from a set of 24 objects by asking questions. Two problems (each with a specific target object) are given with the type of question (for example, deductive) asked by the subject and whether the subject identified the object or not recorded by the test examiner. This test measures reasoning ability and question development (for example, divergent versus convergent).

\section{MEMORY}

\section{Wechsler memory scale revised}

This is a standardised test that includes several subtests which measure short term memory, verbal and non-verbal learning, and immediate and delayed recall, mental control, and attention.

\section{Experimental paired associate test ${ }^{24}$}

Subjects were asked to remember several types of paired items (either word word, word picture, picture word, or picture picture pairs); each pair was presented on a card. Subjects were next given a visual cued recall test using the first item in each pair, followed by a verbal free recall test and an incidental learning task which required subjects to indicate whether each item was presented originally as a picture or a word. This test evaluates structured versus free recall and incidental memory for a stimulus feature.

\section{Hasher frequency monitoring task ${ }^{24}$}

Subjects were read a set of words that they were told to remember. Some of the words were repeated up to seven times. Following the presentation of this list, subjects were asked to recall as many of the items as they could from the original list. Then, subjects were read all the items from the original list and some items that were never presented before and asked to estimate how many times they had heard these words. This test evaluates sensitivity to frequency of presentation under implicit (free recall) and explicit (forced choice) conditions.

\section{Story memory}

Subjects were read two stories and asked to recall as much information as they could about each story following its presentation. The number of story idea units recalled were recorded.

\section{Word fluency}

Subjects were asked to generate words for 90 seconds that belonged to various categories (for example, animals, countries, things you can buy in supermarkets) or began with a specific letter (for example, F, A, S).

MOOD STATE AND PHYSICAL CONDITION 1. Beck depression inventory ${ }^{25}$
This scale was completed by the subject who indicated the number and severity of symptoms associated with depression that they were currently experiencing. A score of 15 or higher is suggestive of at least a mild depression.

\section{Somatisation scale}

This questionnaire is completed by the subject who indicates whether he is currently experiencing unusual symptoms that are typically associated with somatisation.

\section{Neurobehavioural rating scale}

This scale is completed by the test examiner and indicates the severity of behavioural symptoms (for example, depressed effect) observed by the examiner during the test examination. The higher the score per behavioural symptom, the more severe the symptom appears to the observer. There are 29 behavioural symptoms scored on this scale.

\section{Fatigue scale}

Subjects rated their fatigue on an unpublished analogue scale developed by the Center for Disease Control reflecting the degree of fatigue they were experiencing on the day of testing, the month before the testing, and the month before their illness.

These scales and questionnaires are used for screening purposes only. A psychiatric diagnosis requires a formal psychiatric interview which was not completed on any of the patients or controls in conjunction with this evaluation.

\section{STATISTICAL ANALYSES}

Analysis of variance was used for most between group comparisons. Posthoc evaluations used the Scheffe test. Regression analysis was used to determine the effects of age, education, and sex on specific measures. Pearson product moment correlations were computed to evaluate the relationship between performance on related cognitive measures. The error bars in the figures always refer to standard deviations.

\section{Results}

GENERAL INTELLECTUAL AND COGNITIVE FUNCTIONS

There were no significant between group differences in performance on tests of intelligence, reaction time, planning, time perception, or problem solving, with two exceptions. The CFS patients demonstrated greater variability than the controls in the timing of their tapping on the time clock task $(\mathrm{F}(1,33)=4.62, \mathrm{p}<0.03)$ although the overall mean tapping times of the CFS patients and controls were similar. CFS patients solved more problems than did the controls on the Tower of London task $(F(1,33)=$ $4.04, \mathrm{p}<0.05)$. On the other hand, CFS patients tended to make more errors in solving the Tower of London problems $(F(1,30)$ $=7.38, \mathrm{p}<0.01)$. The essentially normal performance of CFS patients on these mea- 
Table 2 Wechsler memory scale-revised

\begin{tabular}{lrr}
\hline & \multicolumn{2}{l}{ Raw scores } \\
\cline { 2 - 3 } Subtests & \multicolumn{1}{c}{ Controls } & \multicolumn{1}{c}{ CFS patients } \\
\hline Information & $13 \cdot 94(0 \cdot 25)$ & $13 \cdot 90(0 \cdot 31)$ \\
Mental Control & $5 \cdot 44(0 \cdot 73)$ & $5 \cdot 15(0 \cdot 93)$ \\
Figural Memory & $7 \cdot 38(1 \cdot 20)$ & $6 \cdot 79(1 \cdot 08)$ \\
Logical Memory I & $29 \cdot 06(5 \cdot 57)$ & $26 \cdot 05(6.92)$ \\
Logical Memory II & $24 \cdot 38(6 \cdot 64)$ & $20 \cdot 50(8 \cdot 10)$ \\
Visual Paired-Associates I & $16 \cdot 00(2 \cdot 73)$ & $14 \cdot 85(3 \cdot 03)$ \\
Visual Paired-Associates II & $5 \cdot 94(0 \cdot 25)$ & $5 \cdot 70(0.66)$ \\
Verbal Paired-Associates I & $20 \cdot 75(3 \cdot 53)$ & $20 \cdot 70(2 \cdot 68)$ \\
Verbal Paired-Associates II & $7 \cdot 69(0 \cdot 60)$ & $7 \cdot 70(0.57)$ \\
Visual Reproduction I & $36 \cdot 50(2 \cdot 37)$ & $34 \cdot 00(3.51)^{\star}$ \\
Visual Reproduction II & $33 \cdot 13(5 \cdot 73)$ & $28 \cdot 50(6 \cdot 34)^{\star}$ \\
\hline
\end{tabular}

The only significant differences between groups occurred on the visual reproduction subtest. The only significant differences between groups occurred on the visual reproduction subtest.

However, CFS patients generally performed
(Means and standard deviations are shown.) the experimental paired associates and Hasher frequency tasks), CFS patient recall and judgment were similar to those of the controls. On the first of two experimental stories, the CFS patients recalled as many story idea units as did the controls. However, following the second story, patients recalled significantly fewer idea units than did controls $(F(1,33)=15 \cdot 26, p<0 \cdot 0004)$. Furthermore, on the experimental paired associates test (see fig 1), CFS patients recalled fewer words in the cued retrieval condition than the controls $(F(1,35)=4.81, p<0.03)$.

Thus, CFS patients appeared to have generally normal memory functioning, yet, paradoxically, they demonstrated specific difficulties in recalling information under conditions of greater semantic structure (for example, cued as opposed to free recall and recall of story propositions as opposed to isolated words) and in reproducing geometric designs. This is the opposite of the common finding in which normal subjects and patients with various types of central nervous system impairments are generally helped by increasing stimulus structure (as seen in story memory or cued recall tasks). Correlational analysis was not particularly helpful in identifying associations between other variables (for example, age, education, degree of fatigue, mood state) and these unusual memory deficits in the CFS patients although verbal IQ was significantly correlated with the number of propositions retrieved on story two $(r=$ $0 \cdot 46$ ), suggesting that the general verbal skills of these patients were partially responsible for their poorer performance.

\section{MOOD STATE AND EFFORT}

There was no difference in the number of depressive symptoms reported by the CFS patients and controls on the Beck depression inventory. Scores from an examiner rating scale showed that about a quarter of the CFS patients demonstrated mild to moderately impaired attention and memory during the evaluation. As expected, CFS patients reported being significantly more fatigued on the day of testing than controls $(F(1,30)=$ $46.37, \mathrm{p}<0.0001)$ and their fatigue on the testing day was compatible with their general level of fatigue throughout the illness. However, there were no differences between the level of pre-illness fatigue in the CFS patients and the current level of fatigue in the controls. Furthermore, there was no relationship between any of these fatigue measures and cognitive performance in the CFS group.

This series of tests demonstrated, then, that CFS patients have selective deficits in memory processing arising against a background of relatively normal cognitive functioning. Curiously, they had greater problems recalling material when the material or retrieval measure was the most structured. There was no relationship between performance on these specific memory measures and mood state, fatigue level, age, or education although verbal intelligence was correlated with story memory performance. 
Discussion

The cumulative results of the pilot and neuropsychological studies indicate that reports of memory impairment dominate the current and past cognitive complaints of patients with CFS. For both the current and past periods, the level of depression correlated directly with the number and severity of memory complaints. Importantly, neither the current or recalled complaints of cognitive or mood problems could be predicted on the basis of any other clinical or laboratory data accrued. On objective neuropsychological testing, we found only a few, selective memory deficits in the CFS patients consistent with their primary complaints on the survey. However, performance on objective memory tests was not correlated with scores on the Beck depression inventory (which were generally within normal limits on the day of testing).

Had we tested patients who were more acutely ill (for example, bedridden patients), we might have observed more severe and widespread cognitive deficits. However, the severity of complaints in our patient cohort was typical of that usually published, was sufficient to permit a diagnosis of CFS according to CDC criteria, and sufficient to render two thirds of them vocationally disabled..$^{146-30}$ It is true that the mean scores of the CFS patient group, while not significantly different from the controls on most measures, were generally slightly lower than the controls. Nevertheless, it is not obvious that this modest difference could fully explain the extent of CFS patient distress over their cognitive and memory functions. Thus, we can conclude with reasonable certainty that the cognitive abilities of CFS patients are generally intact.

There are a few caveats to our findings. Firstly, not all 54 of the respondents in the pilot study were available for cognitive studies. It is possible that those patients who did not complete the survey, and in particular those who did not travel to our laboratories for cognitive testing, were the more seriously disabled patients. Yet two thirds of the participants in the cognitive study, as in the pilot study, were vocationally disabled owing to their chronic fatigue.

Secondly, the Beck depression inventory, while being an excellent self rating index of depression, cannot substitute for a careful psychiatric examination and diagnosis. ${ }^{31}$ Nevertheless, we believe that true depressive symptomatology based on emotional rather than physical symptoms was measured on the surveys. The emotional status scale scores, which reflected the frequency of crying, agitation, and similar behaviours, correlated highly with the Beck depression scale scores. Furthermore, the present finding of a high prevelance of depression is consistent with earlier findings of structured psychiatric interviews conducted in our, and other groups of, CFS patients. ${ }^{8932}$ Even though we found a strong relationship between elevated depressive symptomatology and memory complaints, other factors such as degree of fatigue may also predict memory complaints.
Thirdly, although we found no strong relation between biological measures and current complaints of cognitive problems or mood state abnormalities, we cannot rule out such a relationship. A stronger relation might have been found had we conducted the survey and the medical assessments concurrently. On the other hand, abnormal physical findings are unusual, routine laboratory tests are typically normal, and immunoglobulin levels and viral antibody titres have proved to be remarkably stable over periods of a year or longer in this syndrome. ${ }^{2}$ It is entirely possible that more sophisticated immunological or neuroendocrine tests-for example, not conducted here, would show variation in accord with the symptoms, although objective markers of disease severity, such as these, have yet to be identified and confirmed. ${ }^{33} 34$

CFS patients complain consistently of concentration and memory problems, particularly memory for recently presented information. ${ }^{1429}$ For example, some patients report severe impairment when required to describe a film or television show previously viewed, when recalling conversations from earlier in the day, remembering appointments, or recognising the faces of people recently introduced to them. This level of severity of memory deficits is typical of patients who have suffered a moderate closed head injury. There has been only one other published peer reviewed neuropsychological study of CFS that has used a wide range of objective tests to assess the degree of memory impairment as reported by CFS patients in our pilot study ${ }^{35}$ (but also see the studies by Altay and $S{ }^{3}{ }^{36}{ }^{37}$ ). The results of those studies were similar to ours and the authors described their patients as demonstrating a discrete deterioration of short-term memory. ${ }^{35}$ While the results of our study indicate that CFS patients experience some impairment on selective measures of memory; the severity of this impairment is relatively mild and inconsistent with the severity of complaints on the self report survey. We are unable to identify the cause(s) of the mild memory deficit at present.

Both infections and depression may cause a decrease in cognitive effort. ${ }^{38} 39$ Such a decrease in effort can affect the encoding and recall of new information. Thus, memory problems that result from infection and the inflammatory responses to it could be exacerbated by a concurrent depression. ${ }^{40}$

The findings in this study reinforce the importance of formally testing the memory and mood state symptoms in patients with CFS, since these will be among their most prominent complaints. The fact that these symptoms and deficits appear grossly independent of routine indices of illness severity and fatigue ${ }^{4142}$ and, at least in the pilot survey conducted here, are highly intercorrelated, further complicates the diagnostic examination of CFS patients and of illuminating the pathogenesis of CFS. ${ }^{134-46}$

Regardless of the basis for the cognitive and emotional complaints and mild memory 
impairment in CFS patients, these symptoms cause considerable distress. ${ }^{19}$ With the background provided by this study and a recently completed parallel study of selective attention in CFS, in which patients were shown to have normal visual attentional processes but slowed response execution, ${ }^{47}$ we have developed a more focused testing protocol for continued prospective and objective measurement of neuropsychological complaints in CFS. ${ }^{48} 49$

1 Holmes GP, Kaplan JE, Gantz NM, et al. Chronic fatigue syndrome: a working case definition. Ann Intern Med 1988;108:387-9.

2 Straus SE, et al. Acyclovir treatment of the chronic fatigue syndrome: lack of efficacy in a placebo-controlled trial. N Engl F Med 1988;319:1692-8.

3 Gold D, Bowden R, Sixbey J, et al. Chronic fatigue: a prospective clinical and virologic study. $\mathcal{F A M A} 1990$; 264:48-53.

4 Gosling FG. Before Freud: neuroasthenia and the American Medical Community, 1870-1910. Urbana, Illinois: University of Illinois Press, 1987.

5 Abbey SE, Garfinkel PE. Neurasthenia and chronic fatigue syndrome: the role of culture in the making of a fatigue syndrome: the role of culture in the mat
diagnosis. Am $\mathcal{F}$ Psychiatry 1991;148:1638-46.

6 Mechanic D. Social psychologic factors affecting the presentation of bodily complaints. $N$ Engl $\mathcal{H}$ Med 1972; 286:1132-9.

7 Hickie I, Lloyd A, Wakefield D, et al. The psychiatric status of patients with the chronic fatigue syndrome. $\mathrm{Br} \mathcal{F}$ Psychiatry 1990;156:534-40.

8 Manu P, Lane TJ, Matthews DA. The frequency of chronic fatigue syndrome in patients with symptoms of persistent fatigue. Ann Intern Med 1988;109:554-6.

9 Abbey SE, Garfinkel PE. Chronic fatigue syndrome and the psychiatrist. Can $\mathcal{F}$ Psychiatry 1990;35:625-33.

10 Cohen S, Williamson GM. Stress and infectious disease. Psychol Bull 1991;109:5-24.

11 Cohen S, Tyrrell DAJ, Smith AP. Psychological stress and susceptibility to the common cold. $N$ Engl $f$ Med 1991;325:606-12

12 Kranzler HR, Manu P, Hesselbrock V, et al. Substance use disorders in patients with chronic fatigue. Hosp Comm Psychiatry 1991;42:924-8.

13 Wessely S. Chronic fatigue syndrome. $\mathcal{F}$ Neurol Neurosurg Psychiatry 1991;54:669-71.

14 Straus S. The chronic mononucleosis syndrome. 7 Infect Dis 1988;157:405-12.

15 Edelstein H, Knight RT. Epstein-Barr virus causing encephalitis in an elderly woman. SMF 1989;82:1192-3.

16 Ray C. Chronic fatigue syndrome and depression: conceptual and methodological ambiguities. Psychol Med tual and me

17 Powell R, Dolan R, Wessely S. Attributions and selfesteem in depression and chronic fatigue syndrome. esteem in depression and chro 7 Psychosom Res 1990;34:665-73.

18 Yabuki S, Kazahaya Y, Kubonishi I. Epstein-Barr virus antibodies in neurological diseases. Folia Psychiatry Neurol Fap 1985;39:85-93.

19 Grafman J, Johnson RJ, Scheffers M. Cognitive and mood-state changes in patients with chronic fatigue syndrome. Rev Infect Dis 1991;13(Suppl 1):S45-52.

20 Bennett-Levy J, Powell GE. The subjective memory questionnaire $(S M Q)$ : an investigation into the self-reporting of real-life memory skills. Br 7 Soc Clin Psychol 1980;19: $177-88$.

21 Lezak M. Neuropsychological assessment. 2nd ed. New York: Oxford University Press, 1983.

22 Grafman J, Weingartner $\mathbf{H}$, Newhouse $\mathrm{P}$, et al. Implicit learning in patients with Alzheimer's disease. learning in patients with Al

23 Grafman J, Litvan I, Massaquoi S, et al. Cognitive planning deficit in patients with cerebellar atrophy.
Neurology 1992;42:1493-6.

24 Grafman J, Rao S, Bernardin L, et al. Automatic memory processes in patients with multiple sclerosis. Arch Neurol 1991;48:1072-5.

25 Beck AT. Beck depression inventory: Manual. San Antonio, Texas: Psychological Corporation, 1987.

26 Holland R. Chronic fatigue syndrome. Can Med Ass $\mathcal{f}$ 1989;141:375.

27 Holmes GP, Kaplan JE, Stewart JA, et al. A cluster of patients with a chronic mononucleosis-like syndrome: is Epstein-Barr virus the cause? $\mathscr{f A M A} 1987 ; 257$ : 2297-302.

28 Katz BZ, Andiman WA. Chronic fatigue syndrome. F Pediatr 1988;113:944-7.

29 Straus SE, Tosato G, Armstrong G, et al. Persisting illness and fatigue in adults with evidence of Epstein-Barr virus infection. Ann Intern Med 1985;102:7-16.

30 Jones JF, Ray CG, Minnich LL, et al. Evidence of active Epstein-Barr virus infection in patients with persistent, unexplained illnesses: elevated anti-early antigen antiunexplained illnesses: elevated anti-eart

31 Wells KB, et al. Detection of depressive disorder for patients receiving prepaid or fee-for-service care: results from the medical outcomes study. $\mathscr{F} A M A, 1989 ; 262$ : 3298-302.

32 Kreusi MJP, Dale J, Straus SE. Psychiatric diagnoses in patients who have chronic fatigue syndrome. $\mathcal{f}$ Clin Psychiatr 1989;50:53-6.

33 Klimas NG, Salvato FR, Morgan R, et al. Immunologic abnormalities in chronic fatigue syndrome. $f$ Clin Microbiol 1990;28:1403-10.

34 Demitrack MA, Dale JK, Straus SE, et al. Evidence for impaired activation of the hypothalamic-pituitaryadrenal axis in patients with chronic fatigue syndrome. adrenal axis in patients with chronic fatigue
C Clin Endocrin Metabol 1991;73:1224-34.

35 Riccio M, Thompson C, Wilson B, et al. Neuropsychological and psychiatric abnormalities in myalgic encephalomyelitis: a preliminary report. $\mathrm{Br} \mathcal{f}$ Clin Psychol 1992;31:111-20.

36 Altay HT, Abbey SE, Toner BB, et al. The neuropsychological dimensions of postinfectious neuromyasthenia (chronic fatigue syndrome): a preliminary report. Int $\mathcal{F}$ Psychiatry Med 1990;20:141-9.

37 Smith A. Cognitive changes in myalgic encephalomyelitis. In: Jenkins $\mathrm{R}$, Mowbray JF, eds. Post-viral fatigue symdrome. New York: Wiley, 1991: 179-94.

38 Smith AP, Tyrrell DAJ, Al-Nakib W, et al. Effects and after-effects of the common cold and influenza on human performance. Neuropsychobiology 1989;21:90-3.

39 Weingartner H, Silberman E. Cognitive changes in depression. In: Post R, Ballenger J, eds. Neurobiology of depression. In: Post R, Ballenger J, eds. Neurobiology

40 Blakely AA, Howard RC, Sosich RM, et al. Psychiatric symptoms, personality, and ways of coping in chronic fatigue syndrome. Psychol Med 1991;21:347-62.

41 Monks J. Experiencing symptoms in chronic illness: fatigue in multiple sclerosis. Int Disabil Studies 1989; 11:78-83.

42 White PD. Fatigue and chronic fatigue syndromes. In Bass CM, ed. Somatization: physical symptoms and psychological illness. London: Blackwell Scientific Publications, 1990:104-40.

43 Imboden JB, Canter A, Leighton EC. Convalescence from Influenza. Arch Intern Med 1961;108:393-9.

44 Cluff LE. Medical aspects of delayed convalescence. Rev Infect Dis 1991;13(Suppl 1):S138-40.

45 Kasl SV, Evans AS, Niederman JC. Psychosocial risk factors in the development of infectious mononucleosis. Psychosom Med 1979;41:445-66.

46 Kiecolt-Glaser JK, Kennedy S, Malkoff S, et al. Marital discord and immunity in males. Psychosom Med 1988; 50:213-29.

47 Scheffers M, Johson Jr R, Grafman J, et al. Attention and short-term memory in chronic fatigue syndrome patients: an event-related potential analysis. Neurology [in press].

48 Schluederberg A, Straus SE, Grufferman S. Considerations in the design of studies of chronic fatigue syndrome. RID 1991;13 (Suppl 1):S1-S140.

49 Sharpe MC, Archard LC, Banatvala JE, et al. A reportchronic fatigue syndrome: guidelines for research. $₹ R$ Soc Med 1991;84:118-21. 